\title{
Multiple adhesin proteins on the cell surface of Streptococcus gordonii are involved in adhesion to human fibronectin

\begin{abstract}
Nicholas S. Jakubovics
nick.jakubovics@newcastle.ac.uk
\end{abstract} \\ Correspondence \\ Received 24 June 2009 \\ Revised 29 July 2009 \\ Accepted 30 July 2009

\author{
Nicholas S. Jakubovics, ${ }^{1}$ Jane L. Brittan, ${ }^{2}$ Lindsay C. Dutton ${ }^{2}$ \\ and Howard F. Jenkinson ${ }^{2}$ \\ ${ }^{1}$ School of Dental Sciences, Newcastle University, Framlington Place, Newcastle upon Tyne \\ NE2 4BW, UK \\ ${ }^{2}$ Department of Oral and Dental Sciences, University of Bristol, Lower Maudlin Street, Bristol \\ BS1 2LY, UK
}

\begin{abstract}
Adhesion of bacterial cells to fibronectin (FN) is thought to be a pivotal step in the pathogenesis of invasive infectious diseases. Viridans group streptococci such as Streptococcus gordonii are considered commensal members of the oral microflora, but are important pathogens in infective endocarditis. S. gordonii expresses a battery of cell-surface adhesins that act alone or in concert to bind host receptors. Here, we employed molecular genetic approaches to determine the relative contributions of five known S. gordonii surface proteins to adherence to human FN. Binding levels to $\mathrm{FN}$ by isogenic mutants lacking $\mathrm{Hsa}$ glycoprotein were reduced by $70 \%$, while mutants lacking CshA and CshB fibrillar proteins showed approximately $30 \%$ reduced binding. By contrast, disruption of antigen I/II adhesin genes $\operatorname{ssp} A$ and $s s p B$ in a wild-type background did not result in reduced $\mathrm{FN}$ binding. Enzymic removal of sialic acids from FN led to reduced $S$. gordonii DL1 adhesion (>50\%), but did not affect binding by the hsa mutant, indicating that Hsa interacts with sialic acid moieties on FN. Conversely, desialylation of FN did not affect adherence levels of Lactococcus lactis cells expressing SspA or SspB polypeptides. Complementation of the hsa mutant partially restored adhesion to FN. A model is proposed for FN binding by $S$. gordonii in which $\mathrm{Hsa}$ and CshA/CshB are primary adhesins, and SspA or SspB play secondary roles. Understanding the basis of oral streptococcal interactions with FN will provide a foundation for development of new strategies to control infective endocarditis.
\end{abstract}

\section{INTRODUCTION}

Adhesive interactions between bacterial cell-surface molecules and host proteins or glycoproteins form the first step in colonization by commensal microbes and pathogens alike. In humans, fibronectin (FN) is a major target of bacterial adhesion mechanisms. FN is a high-molecularmass glycoprotein that is present in adults in two forms: soluble FN circulates in the bloodstream and cellular FN is found in the extracellular matrix. Adhesion of bacteria to cellular FN is thought to be a central element in a variety of infectious diseases (Joh et al., 1999). The molecular interactions underlying bacterial binding to FN have been particularly well studied in the Gram-positive pathogens Staphylococcus aureus and Streptococcus pyogenes (SchwarzLinek et al., 2006). These interactions involve recognition of the FN peptide backbone by bacterial adhesins through a tandem $\beta$-zipper motif (Schwarz-Linek et al., 2003).

Abbreviations: FN, fibronectin; sWGA, succinylated wheatgerm agglutinin.
Bacterial infective endocarditis is a rare, but extremely dangerous, infection of the cardiac endothelium usually at the site of the heart valves. Infective endocarditis most commonly affects patients with pre-existing defects or damage to the heart valve tissue, or with prosthetic heart valves. Even with available antibiotic therapies, mortality from infective endocarditis remains at levels approaching $25 \%$ (Sy et al., 2009). Gram-positive cocci account for $>80 \%$ of bacterial infective endocarditis cases, and oral viridans streptococci, including Streptococcus gordonii, Streptococcus sanguinis and Streptococcus mitis, are the most common causative agents of native valve endocarditis (Tomás Carmona et al., 2007). A key step in the pathogenesis of infective endocarditis is the attachment of bacteria to the endocardial lining. Oral streptococci possess a variety of adhesins that recognize host cells and extracellular components, including FN.

The major cell-surface adhesins of $S$. gordonii include antigen I/II (AgI/II) family proteins SspA and SspB (Jakubovics et al., 2005b), cell-surface fibrillar proteins 
CshA and CshB (McNab et al., 1999), sialic-acid-binding protein Hsa/GspB (Bensing \& Sullam, 2002; Takahashi et al., 2002) and amylase-binding proteins $\mathrm{AbpA}$ and $\mathrm{AbpB}$ (Tanzer et al., 2003). Of these, only Hsa and its allelic variant GspB have been directly examined for their roles in endocarditis. Disruption of $h s a$ in S. gordonii DL1 (Challis) or $g s p B$ in S. gordonii M99 significantly reduce virulence in the rat model of endocarditis (Takahashi et al., 2006; Xiong et al., 2008). Thus, Hsa/GspB is an important virulence determinant in the pathogenesis of streptococcal endocarditis.

The identification of streptococcal adhesins involved in interactions with specific host substrates is often complicated by the presence of multiple redundant adhesins on the streptococcal cell surface. Thus, binding of $S$. gordonii to salivary agglutinin glycoprotein gp 340 , an innate defence molecule, involves the concerted activities of SspA, SspB and Hsa (Jakubovics et al., 2005a). Purified SspA and SspB proteins interact with $\mathrm{FN}$ and with $\beta 1$ integrins (Nobbs et al., 2007). Adhesion of S. gordonii cells to FN also appears to involve a number of streptococcal proteins. Adhesins CshA and CshB are required for maximum binding to FN (McNab et al., 1996). However, a double mutant disrupted in genes encoding both CshA and CshB retained approximately $70 \%$ of wild-type levels of FN binding (McNab et al., 1996), indicating that additional adhesins contributed to $\mathrm{FN}$ recognition by $S$. gordonii. S. gordonii FbpA, a homologue of $S$. pyogenes FN-binding protein FBP54, is involved in FN binding primarily through regulation of CshA/CshB expression (Christie et al., 2002). In addition, mutants in the gene encoding peptide methionine sulfoxide reductase, MsrA, are significantly impaired in adhesion to FN (Giomarelli et al., 2006). MsrA is required for the stabilization of bacterial cell-surface adhesins (Wizemann et al., 1996), and therefore loss of this protein probably influences multiple adhesin-receptor interactions. In this study, we aimed to characterize the roles of major adhesins CshA, CshB, SspA, SspB and Hsa in FN adhesion by $S$. gordonii. Isogenic mutants lacking individual adhesins or combinations of adhesins were constructed and tested for their capacities to bind FN. Furthermore, Hsa and AgI/II adhesins SspA and SspB were expressed in adhesion-deficient strains of $S$. gordonii or Lactococcus lactis. The results indicate that CshA/CshB and $\mathrm{Hsa}$ are major $S$. gordonii adhesins for FN, and that AgI/II adhesins SspA and SspB have minor roles. A model to describe the multifaceted nature of FN binding by $S$. gordonii is presented.

\section{METHODS}

Bacterial strains and growth conditions. S. gordonii DL1 (Challis) and isogenic mutants UB645 (cshA31:: cat cshB2::ermAM; identical to OB277), UB1360 (sspAB::aad9), UB1545 (hsa::aphA3) and UB1552 (sspAB::aad9 hsa::aphA3) have been described previously (Heddle et al., 2003; Jakubovics et al., 2005a; McNab et al., 1994). S. gordonii strains were routinely cultured at $37{ }^{\circ} \mathrm{C}$ without shaking in BHY medium containing $\left(1^{-1}\right) 37 \mathrm{~g}$ Brain Heart Infusion (Difco) and
5 g yeast extract. Alternatively, cells were incubated at $37{ }^{\circ} \mathrm{C}$ on solidified medium (BHYN) composed of BHY supplemented with $\left(1^{-1}\right) 5 \mathrm{~g}$ Neo-peptone (Difco) and $15 \mathrm{~g}$ Bacto-agar. For adhesion assays, bacteria were cultured at $37{ }^{\circ} \mathrm{C}$ in TYG medium containing $\left(1^{-1}\right) 10$ g Bacto-tryptone (Difco), 5 g yeast extract, $3 \mathrm{~g} \mathrm{~K}_{2} \mathrm{HPO}_{4}$ and $2 \mathrm{~g}$ D-glucose, and adjusted to $\mathrm{pH} 7.5$ prior to autoclaving. L. lactis MG1363, and plasmid-bearing derivative strains (Jakubovics et al., 2005b) were grown without shaking at $30{ }^{\circ} \mathrm{C}$ in M17 medium containing $5 \mathrm{~g}$ D-glucose $1^{-1}$ (GM17) (Jakubovics et al., 2005b). For maintenance of chromosomal deletion constructs, antibiotics were included at the following concentrations $\left(\mu \mathrm{g} \mathrm{ml}^{-1}\right)$ : chloramphenicol, 5; erythromycin, 2; spectinomycin, 250; kanamycin, 250. Plasmids based on pMSP7517 or pUB1000 were maintained by inclusion of $5 \mu \mathrm{g}$ erythromycin $\mathrm{ml}^{-1}$ in the growth medium.

Genetic manipulations. Routine cloning procedures were carried out in accordance with protocols described by Sambrook et al. (1989). Chromosomal DNA was extracted from streptococci following cell lysis with mutanolysin (Jenkinson, 1987). Transformation of streptococci was performed as described by Haisman \& Jenkinson (1991). S. gordonii UB1927 (cshA31::cat cshB2::ermAM sspAB::aad9) was generated by transforming $S$. gordonii UB645 with chromosomal DNA extracted from S. gordonii UB1360 and selecting for chloramphenicol, erythromycin and spectinomycin resistance. Similarly, S. gordonii UB1928 (cshA31::cat cshB2::ermAM $h s a:: a p h A 3)$ was constructed by introducing chromosomal DNA from S. gordonii UB1545 into S. gordonii UB645 by natural transformation and selecting for chloramphenicol, erythromycin and kanamycin resistance. Transformation of S. gordonii UB1927 with DNA extracted from S. gordonii UB1545, and selection for resistance to four antibiotics (chloramphenicol, erythromycin, spectinomycin and kanamycin) generated S. gordonii UB1929 (cshA31:: cat cshB2:: ermAM sspAB:: aad9 hsa::aphA3).

Determination of adhesion to $\mathbf{F N}$ and fetuin. Human plasma FN (Roche Diagnostics) and bovine fetuin (Sigma-Aldrich) were employed as substrates for adhesion assays. These substrates were diluted in coating buffer $\left(20 \mathrm{mM} \mathrm{Na} \mathrm{CO}_{3}, 20 \mathrm{mM} \mathrm{NaCO}_{3}, \mathrm{pH} 9.3\right)$ and immobilized on the surfaces of Immobilon microtitre plate wells (Nunc) at $4{ }^{\circ} \mathrm{C}$ for $20 \mathrm{~h}$. Wells were washed with PBS, and nonspecific binding sites were blocked with $1 \%(\mathrm{w} / \mathrm{v})$ BSA for $1 \mathrm{~h}$ at $20{ }^{\circ} \mathrm{C}$. Wells were washed with TBSC $(10 \mathrm{mM}$ Tris/HCl, $150 \mathrm{mM}$ $\mathrm{NaCl}, 5 \mathrm{mM} \mathrm{CaCl}_{2}, \mathrm{pH}$ 7.6). For some experiments, immobilized FN or fetuin was treated with 0.001 units sialidase (Clostridium perfringens neuramindase type $\mathrm{X}$ from Sigma-Aldrich) in reaction buffer $\left(0.1 \mathrm{M}\right.$ sodium acetate buffer, $\left.2 \mathrm{mM} \mathrm{CaCl}_{2}, \mathrm{pH} 5.0\right)$ at $37{ }^{\circ} \mathrm{C}$ for $2 \mathrm{~h}$ prior to addition of bacterial cells. Streptococci were cultured at $37^{\circ} \mathrm{C}$ for $20 \mathrm{~h}$ in TYG medium, supplemented where appropriate with $100 \mathrm{ng}$ nisin $\mathrm{ml}^{-1}$. Lactococci were incubated for $20 \mathrm{~h}$ in GM17 medium. Bacterial cells were harvested, washed in TBSC, and adjusted to $\mathrm{OD}_{600}=1.0$ in TBSC (approx. $10^{9}$ cells $\mathrm{ml}^{-1}$ ) using an ATI Unicam UV2 spectrophotometer. Cell suspensions $(0.1 \mathrm{ml})$ were added to microtitre plate wells and incubated at $37{ }^{\circ} \mathrm{C}$ for $2 \mathrm{~h}$. Wells were washed three times with TBSC, and $0.1 \mathrm{ml} 25 \%(\mathrm{v} / \mathrm{v})$ formaldehyde was added to fix bound cells. Plates were incubated at $20{ }^{\circ} \mathrm{C}$ for 15 min, washed three times with PBS and cells were stained by the addition of $0.5 \%(\mathrm{w} / \mathrm{v})$ crystal violet $(0.1 \mathrm{ml})$ and incubation for $1 \mathrm{~min}$ at $20{ }^{\circ} \mathrm{C}$. Wells were washed three times with PBS. Residual dye was dissolved in $50 \mu \mathrm{l} 7 \%(\mathrm{v} / \mathrm{v})$ acetic acid and quantified by measuring $A_{595}$. Numbers of cells adhered were calculated from standard curves relating $A_{595}$ readings to numbers of bacterial cells in microtitre plate wells. Statistical analyses were performed using Student's $t$ test.

Heterologous expression of the S. gordonii hsa gene. For expression of the $S$. gordonii hsa gene under control of the nisin induction system, plasmid pMSP7517 (Hirt et al., 2000) was modified 
as follows. pMSP7517 contains the Enterococcus faecalis prgB gene, encoding aggregation substance, downstream of the nisin-inducible promoter $\mathrm{P}_{\text {nisA }}$. Also included in pMSP7517 are nisK and nisR, encoding the nisin sensor and regulator, respectively, that are required for nisin induction, and the ermAM erythromycin resistance cassette. The hsa gene, and associated ribosome-binding site and transcription terminator element, was amplified from $S$. gordonii DL1 (Challis) using the proofreading polymerase Platinum $p f x$ (Invitrogen) and primers $h s a P f 4$ (5'-TGCTCCCATGGAAATTAAGTAGAGGGGATTACATG-3'; NcoI site underlined) and hsaPr4 (5'TGCACCTCGAGAAAAGCTAGAACATCAAGGACT-3'; XhoI site underlined). The PCR product (6655 bp) was digested with NcoI and XhoI. The $\operatorname{prgB}$ gene was excised from plasmid pMSP7517 by digestion with $\mathrm{NcoI}$ and XhoI. The vector was ligated to the NcoI/ XhoI-digested hsa PCR amplification product, and the resultant plasmid, pMSP- $h s a$, was transformed into E. coli DH5 $\alpha$. The presence of the $h s a$ gene in the correct orientation in pMSP- $h s a$ was confirmed by restriction enzyme digestion analysis and by DNA sequencing. Plasmid pMSP-hsa was extracted from E. coli and transformed into $S$. gordonii hsa mutants UB1545 and UB1552.

Lectin blotting. To monitor the induction of the $h s a$ gene product in S. gordonii UB1545(pMSP-hsa), cells were cultured at $37^{\circ} \mathrm{C}$ for $20 \mathrm{~h}$ in TYG medium. Cultures were diluted 100-fold in fresh TYG. At the early exponential phase of growth $\left(\mathrm{OD}_{600}=0.3\right)$, nisin was added to a concentration of $100 \mathrm{ng} \mathrm{ml}^{-1}$. S. gordonii cell-wall proteins were extracted by incubation with mutanolysin (Jakubovics et al., 2000), and $20 \mu \mathrm{g}$ portions were separated under denaturing conditions on $5 \%$ polyacrylamide gels. Proteins were transferred to Amersham Hybond ECL nitrocellulose membranes (GE Healthcare) at $100 \mathrm{~V}$ for $1.5 \mathrm{~h}$. Membranes were probed with biotin-labelled succinylated wheatgerm agglutinin (sWGA), and detected with $0.2 \mu \mathrm{g}$ peroxidaseconjugated streptavidin $\mathrm{ml}^{-1}$. Densitometric analysis of band intensities was performed using ImageJ software (Collins, 2007).

\section{RESULTS}

\section{Adhesion of $\operatorname{csh} A / B, \operatorname{ssp} A / B$ and hsa mutants to FN}

FN-binding mechanisms have been well-described in the Gram-positive organisms Sta. aureus and S. pyogenes (Schwarz-Linek et al., 2006), and involve recognition of the FN peptide backbone by bacterial cell-surface adhesins. In $S$. pyogenes, several different cell-surface proteins contribute to the overall interaction with FN. We hypothesized that binding of S. gordonii cells to FN may be mediated by a combination of cell-surface adhesins. To test this, FN binding by isogenic mutants lacking specific cell-surface adhesins, or adhesin protein families, was assessed using a microtitre plate adhesion assay (see Methods). In agreement with a previous study ( $\mathrm{McNab}$ et al., 1996), adhesion of wild-type S. gordonii DL1 increased with increasing FN over the range $0.25-5 \mu \mathrm{g}$ FN per well (Fig. 1). Between 1 and $5 \mu \mathrm{g}$ FN per well, there was only a small $(<20 \%)$ increase in the number of cells bound. Adhesion of isogenic mutants lacking $\operatorname{csh} A$ and $\operatorname{csh} B$ genes to $\mathrm{FN}$ was up to $28 \%$ lower than wild-type at all FN concentrations tested. Adhesion of the $\operatorname{csh} A / \operatorname{csh} B$ mutant to 0.5 or $1.0 \mu \mathrm{g}$ FN was significantly reduced $(P<0.05)$ compared with wild-type. At higher concentrations of FN $(2.5$ or $5 \mu \mathrm{g})$, the reduction in binding of the $\operatorname{csh} A / \operatorname{csh} B$ mutant compared with wild-type was not

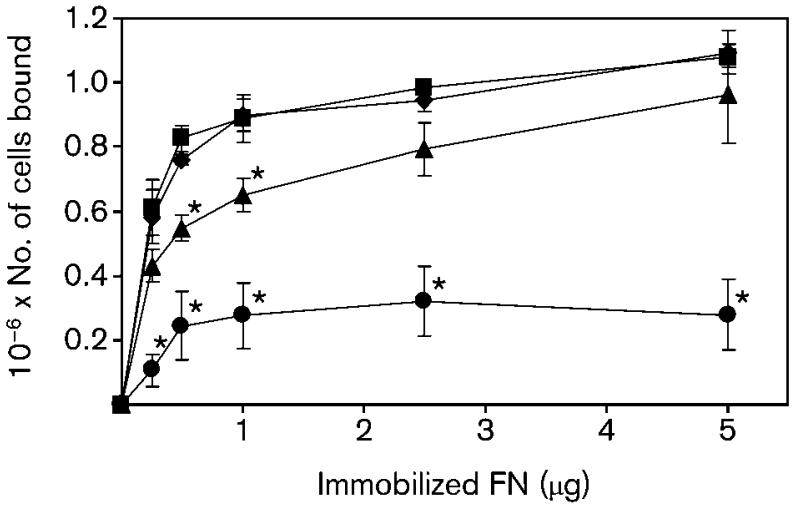

Fig. 1. FN binding by $S$. gordonii DL1 $(\boldsymbol{})$ and isogenic mutants UB1360 $\Delta(\operatorname{ssp} A \operatorname{ssp} B)(\boldsymbol{\square}), \operatorname{UB645} \Delta(\operatorname{csh} A \operatorname{csh} B)(\boldsymbol{\Delta})$, and UB1545 $\Delta$ ssa (O). FN was immobilized on the surface of microtitre wells and incubated in the presence of bacteria. After washing, bound bacterial cells were quantified by staining with crystal violet (see Methods). Means \pm SEM from three independent experiments are shown. Values that were significantly lower $(P<0.05)$ than adhesion levels of $S$. gordonii DL1 at the equivalent FN concentration are marked with an asterisk above and to the right of the data point.

statistically significant ( $P>0.05)$ (Fig. $1 ; \mathrm{McNab}$ et al., 1996). Binding of the $h s a$ mutant to FN was reduced by between 66 and $82 \%$ compared with wild-type at all concentrations tested, and these differences were all statistically significant $(P<0.05)$. By contrast, disruption of AgI/II genes $s s p A / s s p B$ did not result in reduced FN binding levels compared with the isogenic wild-type progenitor (Fig. 1).

\section{Effects of combined cell-surface adhesin gene disruptions on adhesion to $\mathrm{FN}$ and fetuin}

To assess whether cell-surface adhesins of S. gordonii are functionally redundant for FN binding, isogenic mutants were constructed in which multiple proteins were disrupted. All combinations of mutants lacking functional $\operatorname{csh} A / \operatorname{csh} B, \operatorname{ssp} A / \operatorname{ssp} B$ and $h s a$ genes were produced, and adhesion of wild-type $S$. gordonii DL1 and mutant strains to FN $(2.5 \mu \mathrm{g})$ was assessed (Fig. 2a). At this FN concentration there was reduced binding of the $\operatorname{csh} A / \operatorname{csh} B$ mutant to FN, but this was not statistically significant. Similarly, in strains mutated in $s s p A / \operatorname{ssp} B$ or in $h s a$ genes, disruption of $\operatorname{csh} A / \operatorname{csh} B$ resulted in reduced binding to $\mathrm{FN}$ compared with strains bearing intact $\operatorname{csh} A$ and $\operatorname{csh} B$ genes. However, these reductions did not reach statistical significance (Fig. 2a, for example compare UB1927 with UB1360). It seems, therefore, that significantly reduced FN $(1 \mu \mathrm{g})$ binding resulting from disruption of $\operatorname{csh} A$ and $\operatorname{csh} B$ (shown in Fig. 1) was not so evident with higher FN concentrations utilized for the results shown in Fig. 2(a).

Disruption of $h s a$ significantly $(P<0.05)$ reduced adhesion of $S$. gordonii to FN in the presence or absence of intact 

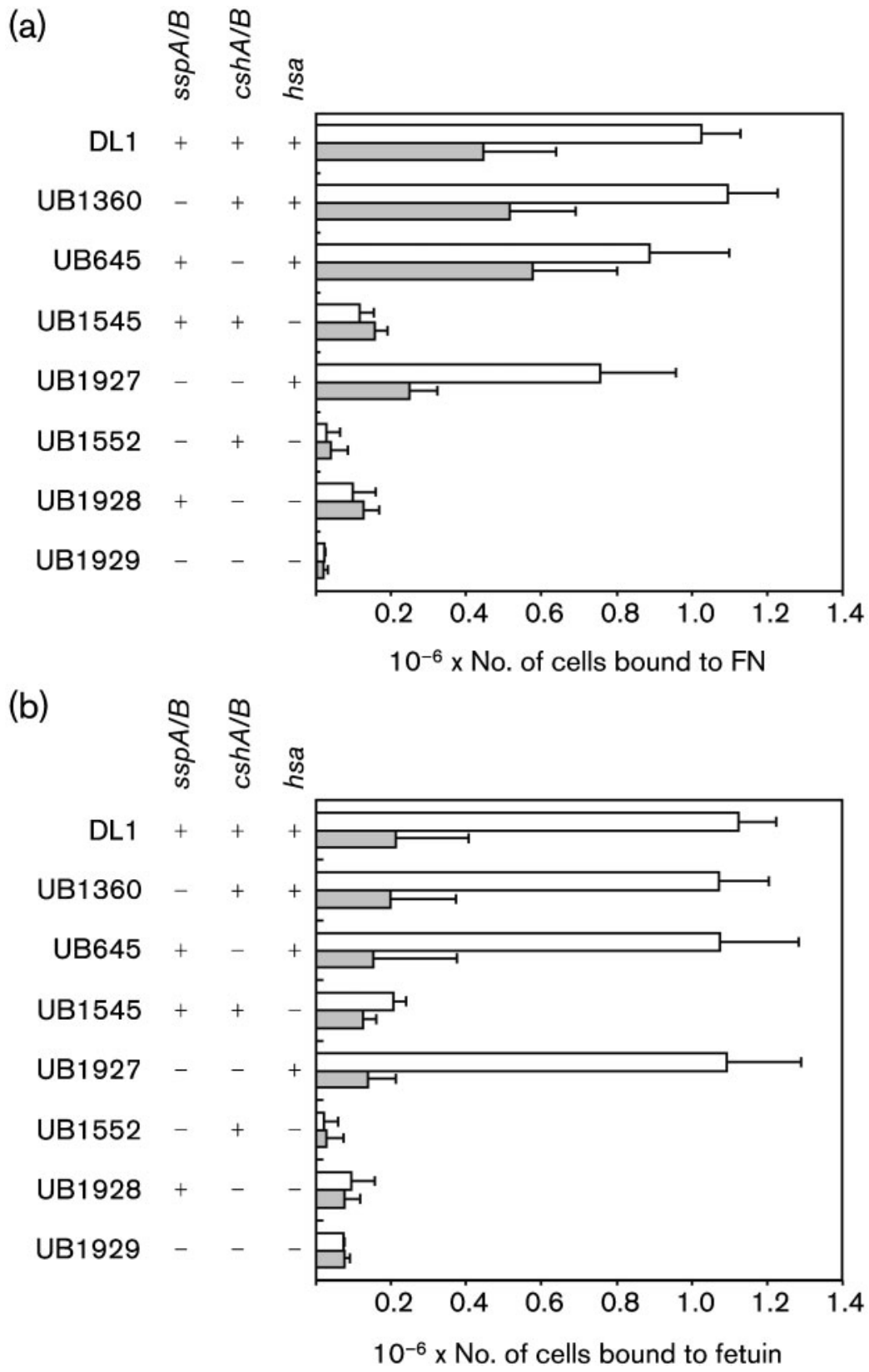

Fig. 2. FN (a) and fetuin (b) adhesion by $S$. gordonii DL1 and isogenic mutants lacking different combinations of cell-surface adhesins. Binding was measured to untreated (white bars) or sialidase-treated (shaded bars) substrate. Bars represent mean values \pm SEM from four independent experiments.

$\operatorname{ssp} A / \operatorname{ssp} B$ or $c \operatorname{sh} A / \operatorname{csh} B$ genes (Fig. 2a). When either $\operatorname{csh} A$ / $\operatorname{csh} B$ or $h s a$ genes were knocked out, reduced levels of adhesion were observed for strains mutated in AgI/II protein genes $s s p A / s s p B$ compared with strains possessing functional $s s p A / s s p B$ (Fig. 2a; compare UB1927 with UB645, UB1929 with UB1928, and UB1552 with UB1545). Although none of these differences were statistically significant, they were consistent and indicated that AgI/II proteins might have secondary roles in influencing adhesion to FN.

\section{Hsa protein interacts with FN}

$\mathrm{FN}$ is a glycoprotein containing approximately $1.2 \%$ sialic acids (Carsons et al., 1987). Substrate interactions by $S$. gordonii Hsa are dependent upon recognition of sialic acids (Takamatsu et al., 2005). We reasoned, therefore, that recognition of FN by $S$. gordonii may involve sialic acid moieties on the termini of FN carbohydrate chains. To test this, FN was treated with sialidase (neuraminidase) prior to the addition of bacterial cells. Adhesion of wild-type $S$. gordonii DL1 was reduced $>50 \%$ following sialidase treatment of FN (Fig. 2a). Similarly, FN binding by sspA/ $\operatorname{ssp} B$ and $\operatorname{csh} A / \operatorname{csh} B$ mutants containing intact hsa (strains UB1360, UB645 and UB1927) was reduced 30-60\% by sialidase treatment. Adhesion of hsa mutant strains UB1545, UB1552, UB1928 and UB1929 to FN was low and was not further reduced by incubation of $\mathrm{FN}$ with sialidase (Fig. 2a). 
The above data indicated that Hsa is a major adhesin for FN. We have previously demonstrated that Hsa protein levels are not affected by disruption of AgI/II protein genes $s s p A / s s p B$ (Jakubovics et al., 2005a). To ensure that disruption of $\operatorname{csh} A / \operatorname{csh} B$ did not modulate expression of Hsa, cell-surface proteins were extracted from $S$. gordonii DL1 and S. gordonii UB645. Serial twofold dilutions were applied to nitrocellulose using a dot blotter, and the membrane was probed with sWGA, which specifically recognizes Hsa glycoprotein in S. gordonii cell-wall extracts (Jakubovics et al., 2005a). Levels of Hsa expression were not changed by disruption of $\operatorname{csh} A / \operatorname{csh} B$ in strain UB645 (data not shown). To further test that Hsa function was not affected by deletion of cell-wall protein genes $\operatorname{csh} A, \operatorname{csh} B$, $\operatorname{ssp} A$ and $\operatorname{ssp} B$, adhesion of $S$. gordonii cell-surface adhesin mutants to bovine fetuin was assessed. Fetuin is a heavily glycosylated protein that has been used as a model substrate for Hsa binding (Takamatsu et al., 2005). S. gordonii DL1 adhered to fetuin in a dose-dependent manner (Fig. 2b and data not shown). Pre-treatment of fetuin with sialidase reduced binding by $>80 \%$. Levels of fetuin binding by mutant $S$. gordonii strains UB1360, UB645 and UB1927, lacking SspA/SspB and/or CshA/ CshB, were almost identical to wild-type binding levels (Fig. 2b). In each case, adhesion of these mutants was reduced $>80 \%$ following sialidase treatment of the fetuin. In contrast, binding of $S$. gordonii UB1545 hsa to fetuin was extremely low ( $15 \%$ of wild-type binding), and was not further reduced by desialylation of the fetuin substrate. Fetuin binding by isogenic mutants lacking Hsa in addition to SspA/SspB and/or CshA/CshB (strains UB1552, UB1928 and UB1929) was barely detectable. These data indicate that Hsa function, in terms of adhesion to fetuin, was not impaired by disruption of SspA/SspB and/or CshA/CshB.

\section{Complementation of the hsa knockout using a nisin-inducible promoter construct}

To examine further the role of Hsa in adhesion to FN, $h s a$ was cloned downstream of a nisin-inducible promoter (Bryan et al., 2000) to create plasmid pMSP-hsa, and introduced into S. gordonii UB1545 hsa and S. gordonii UB1552 hsa sspAB. Expression of Hsa in $S$. gordonii UB1545(pMSP-hsa) was monitored following addition of nisin by lectin blotting using biotinylated sWGA. The Hsa glycoprotein is difficult to extract and forms a diffuse band on SDS-PAGE (Takahashi et al., 2002; Takahashi et al., 2004). An sWGA-positive band $(>250 \mathrm{kDa})$ was detected in cell-wall extracts of $S$. gordonii DL1, and this was barely visible in the complemented mutant prior to nisin addition (Fig. 3a). Within $3 \mathrm{~h}$ after addition of nisin to $S$. gordonii UB1545(pMSP-hsa) culture, Hsa expression was greater than twofold higher than the initial level. Hsa expression continued to increase for $7 \mathrm{~h}$ after induction (Fig. 3a, b). Adhesion to FN was partially restored by expression of $h s a$ from plasmid pMSP-hsa in S. gordonii UB1545 (Fig. 3c). In contrast, there was no difference in FN adhesion between $S$. gordonii UB1545 and S. gordonii UB1545(pMSP7517), expressing the 'empty' vector (data not shown). Binding levels of S. gordonii UB1552(pMSP-hsa) to sialidase-treated FN and untreated FN were similar, and were both significantly greater than the $h s a / s s p A / s s p B$ mutant $S$. gordonii UB1552 (Fig. 3c). These data indicate that expression of $\mathrm{Hsa}$ increases binding to $\mathrm{FN}$, and also to asialo-FN in the absence of SspA and SspB. The increase in binding of $S$. gordonii UB1552 to asialo-FN upon complementation with plasmid pMSP-hsa was unexpected, since Hsa-mediated adhesion is thought to be highly specific for sialic acid residues (Takamatsu et al., 2005). Prolonged incubation in the presence of the inducer nisin led to slightly higher levels of Hsa in strains bearing plasmid pMSP-hsa than in strains with a chromosomal $h s a$ gene (Fig. $3 a$ and $b$ ). It is possible that in the absence of SspA or SspB, increased levels of Hsa promote adhesion of S. gordonii to non-sialic acid carbohydrates on FN or to the FN backbone. Alternatively, the altered expression levels of cell-surface adhesins in this strain may have led to gross changes in cell-wall structure. By comparison, expression of Hsa from plasmid pMSP-hsa in S. gordonii UB1545 or in S. gordonii UB1552 partially restored binding to fetuin, but not to asialofetuin (data not shown).

\section{Heterologous expression of SspA/SspB in L. lactis increases adhesion to FN}

The results presented above indicated that AgI/II proteins of $S$. gordonii may have more minor roles in adhesion to FN compared with the adhesins Hsa and CshA/CshB (see Fig. 2). Heterologous expression systems have previously been employed to assess the functions of streptococcal AgI/ II proteins in the absence of other streptococcal adhesins (Holmes et al., 1998; Jakubovics et al., 2005b). To investigate the potential of $S$. gordonii AgI/II adhesins SspA and SspB to bind FN, we expressed these proteins independently on the cell surface of L. lactis. Adhesion of $L$. lactis expressing SspA or SspB to FN was significantly $(P<0.05)$ increased compared with the wild-type parent strain L. lactis MG1363 (Fig. 4), confirming that these proteins bind to FN. Sialidase treatment of FN did not significantly affect adherence levels, suggesting that the SspA and SspB proteins do not recognize terminal sialic acid moieties. Although SspA and SspB polypeptides interact with FN, they do not seem to be the major cellsurface adhesins mediating adherence to FN.

\section{DISCUSSION}

The above data demonstrate that $S$. gordonii utilizes several different cell-surface adhesins for recognition of human plasma FN. The mechanism of FN binding by S. gordonii has clear differences from the well-characterized interactions between $S$. pyogenes or Sta. aureus and FN. For example, soluble FN inhibits adherence of $S$. pyogenes to immobilized FN, whereas soluble-phase FN enhances binding of $S$. gordonii to immobilized host substrates, 
Time (h) after addition of nisin to

S. gordonii UB1545(pMSP-hsa)

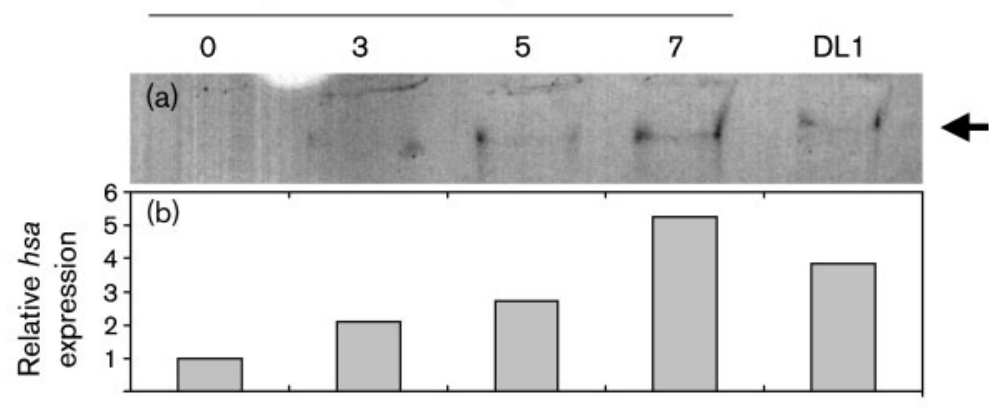

(c)
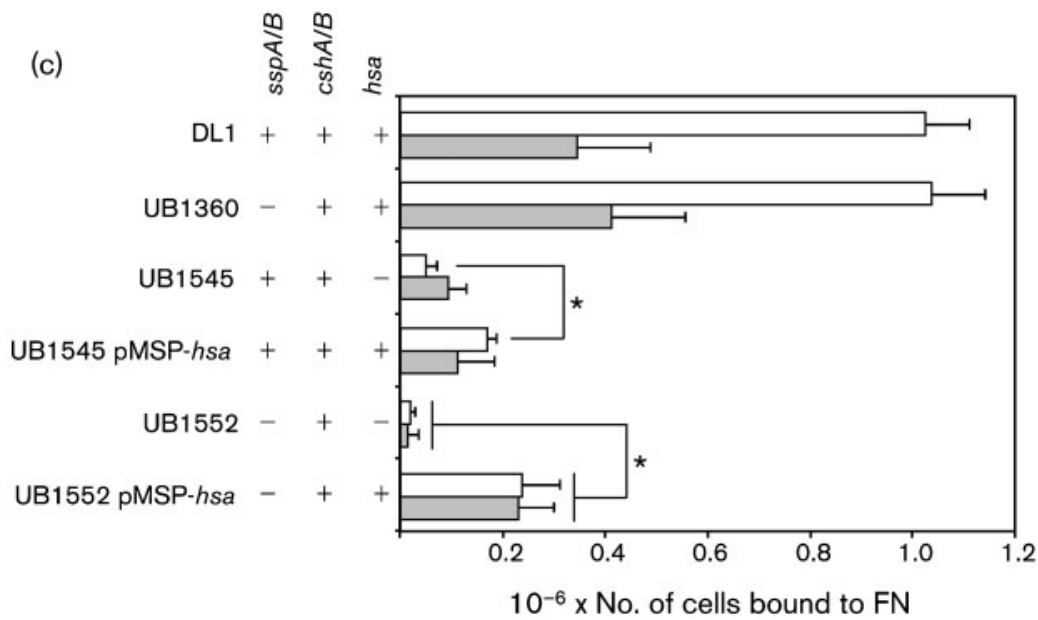

Fig. 3. Effect of Hsa complementation on adhesion of $S$. gordonii to FN. The expression of Hsa from pMSP-hsa was monitored by lectin blotting (a). Cell-wall proteins were extracted from S. gordonii UB1545(pMSP$h s a)$ at different times following induction of $h s a$ expression by addition of nisin to the culture. Equal amounts $(20 \mu \mathrm{g})$ of proteins were separated by PAGE and blotted onto nitrocellulose. Membranes were probed with sWGA, which specifically recognizes $\mathrm{Hsa}$, and a single band was detected at $>250 \mathrm{kDa}$ in each lane (arrow). Extracts from S. gordonii DL1 were included for comparison. The amount of sWGA-reactive protein in each lane was quantified by densitometry (b). (c) Adhesion of S. gordonii strains to immobilized $\mathrm{FN}$ was determined by staining with crystal violet, and mean values \pm SEM from three independent experiments are shown. Significant differences between adhesion of UB1545 and UB1545(pMSP-hsa) to untreated FN, and between UB1552 and UB1552(pMSP-hsa) to untreated (white bars) or sialidase-treated (shaded bars) FN (Student's $t$-test, $P<0.05$ ), are indicated by asterisks. including gelatin, FN and collagen (Lowrance et al., 1988). The major interactions between S. pyogenes or Sta. aureus and FN involve recognition of the FN peptide backbone by bacterial cell-surface adhesins (Schwarz-Linek et al., 2003). We found that adhesion of S. pyogenes A40 or Sta. aureus Newman to FN was not affected by pre-treating FN with sialidase (neuraminidase; data not shown). By contrast, binding of S. gordonii was reduced $>50 \%$ following

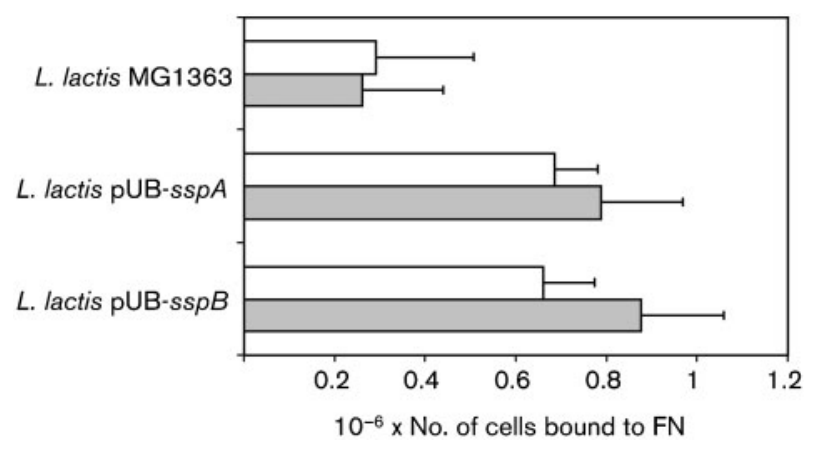

Fig. 4. $F N$ adhesion by $L$. lactis $M G 1363$ and derivative strains expressing Agl/ll polypeptides SspA or SspB from S. gordonii. Binding to untreated (white bars) or sialidase-treated (shaded bars) FN was measured. Mean values \pm SEM from four independent experiments are shown. desialylation of the FN (Fig. 2a). Thus, S. gordonii recognizes and binds to both the peptide backbone and the carbohydrate decorations on FN.

The genetic basis of FN adhesion by $S$. gordonii has been the subject of several investigations. There is strong evidence that CshA/CshB fibrils on the streptococcal cell surface mediate $\mathrm{FN}$ binding. Inactivation of the genes encoding CshA and CshB reduces the ability of S. gordonii to bind immobilized FN by approximately $30-40 \%$ (Fig. 1; $\mathrm{McNab}$ et al., 1996). Antibodies against the $\mathrm{N}$-terminal region of CshA polypeptide inhibit binding of $S$. gordonii to FN (McNab et al., 1996). Moreover, heterologous expression of CshA on the cell surface of E. faecalis promoted adhesion of cells to FN (McNab et al., 1999). More recently, Giomarelli et al. (2006) demonstrated that S. gordonii mutants lacking $\mathrm{Hsa}$ or the AgI/II family proteins SspA and SspB were impaired in their abilities to bind FN. Similarly, we have confirmed that an $h s a$ knockout mutant of S. gordonii DL1 (Challis) has a significantly reduced capacity to bind FN (Fig. 1). However, in contrast to Giomarelli et al. (2006), we were unable to find any reduction in FN binding in a mutant lacking SspA and SspB. Single mutants lacking either SspA or SspB alone were also unimpaired in adhesion to $\mathrm{FN}$ (data not shown). It is possible that our laboratory stock of $S$. gordonii DL1 (Challis) has minor differences in cellsurface composition from the variant used by Giomarelli $e t$ 
al. (2006). Nevertheless, we found some evidence that, in the absence of FN adhesins CshA/CshB and/or Hsa, FN binding was promoted by SspA and SspB (Fig. $2 \mathrm{a}$ and Fig. 4). We propose that $\mathrm{AgI} / \mathrm{II}$ proteins play a secondary role in FN binding by $S$. gordonii. FN binding by AgI/II proteins may be of greater importance for streptococci that produce a more restricted range of cell-surface adhesins. Thus, disruption of the genes encoding AgI/II proteins from Streptococcus intermedius or Streptococcus mutans reduces binding to FN by approximately 75 or $20 \%$, respectively (Petersen et al., 2002).

Based on the data presented here and in previous reports, we propose a model for FN binding by S. gordonii in which CshA/CshB and Hsa polypeptides are primary adhesins and $\mathrm{AgI} / \mathrm{II}$ proteins SspA/SspB are secondary components of the interaction. This model is illustrated in Fig. 5. Altogether, nine glycosylation sites have been described in human plasma FN, seven of which are N-linked and two are O-linked (Tajiri et al., 2005). Both N-linked and Olinked carbohydrates contain terminal sialic acid residues, and these are predicted to be the sites of interaction with $S$. gordonii Hsa. Similarly, Hsa interacts with carbohydrate structures in N-linked and O-linked glycans of the platelet receptor GPIb $\alpha$ (Takamatsu et al., 2005). Hsa accounted for the total interaction of $S$. gordonii with sialic acids; there was no indication that other adhesins bound sialic acids on either FN or fetuin. It is postulated that CshA and CshB polypeptides bind the peptide backbone of FN. Adhesion by SspA and SspB may also involve recognition of FN peptide structures. However, the crystal structure of a homologous AgI/II protein from S. mutans contains a

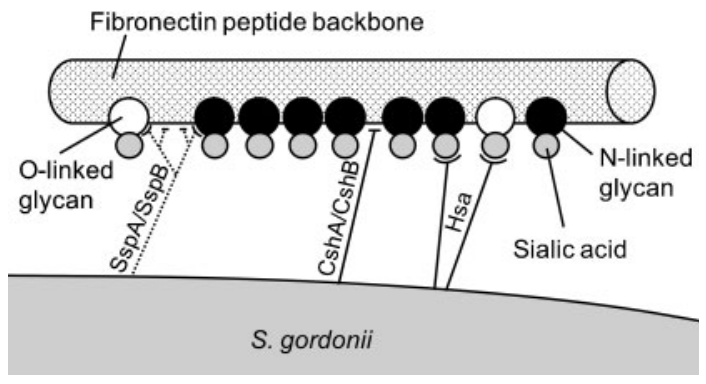

Fig. 5. Model to describe the adhesion interactions between $S$. gordonii DL1 and FN. FN is represented as a polypeptide chain (cylinder) with attached O-linked (white circles) or N-linked (black circles) glycans (based on data from Tajiri et al., 2005). Glycans may contain terminal sialic acid residues (grey circles) that are recognized by S. gordonii Hsa. S. gordonii fibrillar adhesins CshA and $\mathrm{CshB}$ also interact with $\mathrm{FN}$ by recognizing specific peptide structures in the FN backbone. Secondary interactions occur between S. gordonii SspA and SspB and FN that are difficult to observe in the presence of Hsa or CshA/CshB. SspA and SspB adhesins may recognize peptide structures or carbohydrate moieties attached to the FN backbone. carbohydrate-binding fold (Troffer-Charlier et al., 2002), and it is possible, therefore, that SspA and SspB bind to sialidase-insensitive carbohydrate decorations on FN.

Interactions of Hsa with substrates are dependent upon post-translational processing and targeting to the cell surface. Secretion of Hsa requires the secondary secretion system encoded by secA2 and secY2 (Bensing \& Sullam, 2002; Bensing \& Sullam, 2009). S. gordonii Challis has been stored in the laboratory for over 70 years, and at least one clonal line, S. gordonii $\mathrm{CH} 1$, has acquired a point mutation in the $\sec A 2$ gene that prevents the proper secretion of Hsa (Bensing et al., 2004). In contrast to S. gordonii DL1 (Challis), no binding of $S$. gordonii $\mathrm{CH} 1$ (Challis) to immobilized FN was observed (data not shown). To assess the role of $\sec A 2$, a knockout mutant was constructed in which the secA2 gene of $S$. gordonii DL1 (Challis) was disrupted by allelic exchange mutagenesis. This strain did not bind FN to any appreciable level (data not shown). Unfortunately, despite several attempts, and despite previous reports of $\sec A 2$ complementation in the literature (Bensing et al., 2004), we were unable to produce a construct for functional SecA2 protein expression. Therefore, at present it cannot be confirmed that the secA2 mutation in $\mathrm{S}$. gordonii $\mathrm{CH} 1$ (Challis) is the sole cause of the inability of this strain to bind FN. It is possible that co-ordinated $h s a$ and $\sec A 2$ expression may be essential for the optimal function of the Hsa adhesin. This would explain why expression of $h s a$ from a plasmid in $S$. gordonii UB1545(pMSP-hsa) did not fully complement the defective adhesion of the hsa mutant $S$. gordonii UB1545 to FN (Fig. 3c).

In summary, the data presented here reveal a mechanism of FN binding in S. gordonii that involves multiple cell-surface adhesins Hsa, CshA, CshB, SspA and SspB. We demonstrate that bacterial adhesion to $\mathrm{FN}$ is dependent upon recognition of the carbohydrate decorations attached to the FN backbone. This observation calls into question other reports on FN binding by bacteria, where it has often been assumed that bacterial adhesins recognize the FN peptide backbone. Carbohydrate structures on FN may, for example, form receptors for adhesins such as $S$. pyogenes GAPDH, Fbp54 or Shr that do not possess $\beta$-zipperforming domains. Interactions between S. gordonii and FN carbohydrates are primarily mediated by the sialic-acidbinding glycoprotein $\mathrm{Hsa}$, orthologues of which are expressed by strains of $S$. gordonii and $S$. sanguinis (Plummer \& Douglas, 2006; Takamatsu et al., 2006). Genes encoding homologues of CshA, CshB, SspA and SspB are present in the genome sequence of $S$. sanguinis SK36 (Xu et al., 2007), and AgI/II proteins (SspA/SspB homologues) are found in strains of $S$. mutans, S. oralis, $S$. sobrinus and $S$. intermedius. Thus it would be expected that combinations of these functions might confer abilities to bind FN by these other organisms. Understanding these FN interactions better may lead to new molecules that would counteract streptococcal infective endocarditis. 


\section{ACKNOWLEDGEMENTS}

We thank Angela Nobbs and Nicklas Strömberg for helpful discussions. We are very grateful to Gary M. Dunny for supplying plasmid pMSP7517, and to the Netherlands Dairy Research Institute group for their work in developing the nisin induction system. This work was supported by a grant from the Wellcome Trust (grant 064832) awarded to H. F. J.

\section{REFERENCES}

Bensing, B. A. \& Sullam, P. M. (2002). An accessory sec locus of Streptococcus gordonii is required for export of the surface protein GspB and for normal levels of binding to human platelets. Mol Microbiol 44, 1081-1094.

Bensing, B. A. \& Sullam, P. M. (2009). Characterization of Streptococcus gordonii SecA2 as a paralogue of SecA. J Bacteriol 191, 3482-3491.

Bensing, B. A., Lopez, J. A. \& Sullam, P. M. (2004). The Streptococcus gordonii surface proteins GspB and Hsa mediate binding to sialylated carbohydrate epitopes on the platelet membrane glycoprotein Ib $\alpha$. Infect Immun 72, 6528-6537.

Bryan, E. M., Bae, T., Kleerebezem, M. \& Dunny, G. M. (2000). Improved vectors for nisin-controlled expression in Gram-positive bacteria. Plasmid 44, 183-190.

Carsons, S., Lavietes, B. B., Slomiany, A., Diamond, H. S. \& Berkowitz, E. (1987). Carbohydrate heterogeneity of fibronectins. Synovial fluid fibronectin resembles the form secreted by cultured synoviocytes but differs from the plasma form. J Clin Invest 80, 13421349.

Christie, J., McNab, R. \& Jenkinson, H. F. (2002). Expression of fibronectin-binding protein FbpA modulates adhesion in Streptococcus gordonii. Microbiology 148, 1615-1625.

Collins, T. J. (2007). ImageJ for microscopy. Biotechniques 43, 25-30. Giomarelli, B., Visai, L., Hijazi, K., Rindi, S., Ponzio, M., lannelli, F., Speziale, P. \& Pozzi, G. (2006). Binding of Streptococcus gordonii to extracellular matrix proteins. FEMS Microbiol Lett 265, 172-177.

Haisman, R. J. \& Jenkinson, H. F. (1991). Mutants of Streptococcus gordonii Challis over-producing glucosyltransferase. J Gen Microbiol 137, 483-489.

Heddle, C., Nobbs, A. H., Jakubovics, N. S., Gal, M., Mansell, J. P., Dymock, D. \& Jenkinson, H. F. (2003). Host collagen signal induces antigen I/II adhesin and invasin gene expression in oral Streptococcus gordonii. Mol Microbiol 50, 597-607.

Hirt, H., Erlandsen, S. L. \& Dunny, G. M. (2000). Heterologous inducible expression of Enterococcus faecalis pCF10 aggregation substance Asc10 in Lactococcus lactis and Streptococcus gordonii contributes to cell hydrophobicity and adhesion to fibrin. J Bacteriol 182, 2299-2306.

Holmes, A. R., Gilbert, C., Wells, J. M. \& Jenkinson, H. F. (1998). Binding properties of Streptococcus gordonii SspA and SspB (antigen I/ II family) polypeptides expressed on the cell surface of Lactococcus lactis MG1363. Infect Immun 66, 4633-4639.

Jakubovics, N. S., Smith, A. W. \& Jenkinson, H. F. (2000). Expression of the virulence-related Sca $\left(\mathrm{Mn}^{2+}\right)$ permease in Streptococcus gordonii is regulated by a diphtheria toxin metallorepressor-like protein ScaR. Mol Microbiol 38, 140-153.

Jakubovics, N. S., Kerrigan, S. W., Nobbs, A. H., Strömberg, N., van Dolleweerd, C. J., Cox, D. M., Kelly, C. G. \& Jenkinson, H. F. (2005a). Functions of cell surface-anchored antigen I/II family and Hsa polypeptides in interactions of Streptococcus gordonii with host receptors. Infect Immun 73, 6629-6638.
Jakubovics, N. S., Strömberg, N., van Dolleweerd, C. J., Kelly, C. G. \& Jenkinson, H. F. (2005b). Differential binding specificities of oral streptococcal antigen I/II family adhesins for human or bacterial ligands. Mol Microbiol 55, 1591-1605.

Jenkinson, H. F. (1987). Novobiocin-resistant mutants of Streptococcus sanguis with reduced cell hydrophobicity and defective in coaggregation. J Gen Microbiol 133, 1909-1918.

Joh, D., Wann, E. R., Kreikemeyer, B., Speziale, P. \& Höök, M. (1999). Role of fibronectin-binding MSCRAMMs in bacterial adherence and entry into mammalian cells. Matrix Biol 18, 211-223.

Lowrance, J. H., Hasty, D. L. \& Simpson, W. A. (1988). Adherence of Streptococcus sanguis to conformationally specific determinants in fibronectin. Infect Immun 56, 2279-2285.

McNab, R., Jenkinson, H. F., Loach, D. M. \& Tannock, G. W. (1994). Cell-surface-associated polypeptides CshA and CshB of high molecular mass are colonization determinants in the oral bacterium Streptococcus gordonii. Mol Microbiol 14, 743-754.

McNab, R., Holmes, A. R., Clarke, J. M., Tannock, G. W. \& Jenkinson, H. F. (1996). Cell surface polypeptide CshA mediates binding of Streptococcus gordonii to other oral bacteria and to immobilized fibronectin. Infect Immun 64, 4204-4210.

McNab, R., Forbes, H., Handley, P. S., Loach, D. M., Tannock, G. W. \& Jenkinson, H. F. (1999). Cell wall-anchored CshA polypeptide (259 kilodaltons) in Streptococcus gordonii forms surface fibrils that confer hydrophobic and adhesive properties. J Bacteriol 181, 3087-3095.

Nobbs, A. H., Shearer, B. H., Drobni, M., Jepson, M. A. \& Jenkinson, H. F. (2007). Adherence and internalization of Streptococcus gordonii by epithelial cells involves $\beta 1$ integrin recognition by SspA and SspB (antigen I/II family) polypeptides. Cell Microbiol 9, 65-83.

Petersen, F. C., Assev, S., van der Mei, H. C., Busscher, H. J. \& Scheie, A. A. (2002). Functional variation of the antigen I/II surface protein in Streptococcus mutans and Streptococcus intermedius. Infect Immun 70, 249-256.

Plummer, C. \& Douglas, C. W. (2006). Relationship between the ability of oral streptococci to interact with platelet glycoprotein Ib $\alpha$ and with the salivary low-molecular-weight mucin, MG2. FEMS Immunol Med Microbiol 48, 390-399.

Sambrook, J., Fritsch, E. F. \& Maniatis, T. (1989). Molecular Cloning: a Laboratory Manual, 2nd edn. Cold Spring Harbor, NY: Cold Spring Harbor Laboratory.

Schwarz-Linek, U., Werner, J. M., Pickford, A. R., Gurusiddappa, S., Kim, J. H., Pilka, E. S., Briggs, J. A., Gough, T. S., Höök, M. \& other authors (2003). Pathogenic bacteria attach to human fibronectin through a tandem $\beta$-zipper. Nature 423, 177-181.

Schwarz-Linek, U., Höök, M. \& Potts, J. R. (2006). Fibronectin-binding proteins of Gram-positive cocci. Microbes Infect 8, 2291-2298.

Sy, R. W., Chawantanpipat, C., Richmond, D. R. \& Kritharides, L. (2009). Development and validation of a time-dependent risk model for predicting mortality in infective endocarditis. Eur Heart J, Mar 28 [Epub ahead of print].

Tajiri, M., Yoshida, S. \& Wada, Y. (2005). Differential analysis of sitespecific glycans on plasma and cellular fibronectins: application of a hydrophilic affinity method for glycopeptide enrichment. Glycobiology 15, 1332-1340.

Takahashi, Y., Konishi, K., Cisar, J. O. \& Yoshikawa, M. (2002). Identification and characterization of $h s a$, the gene encoding the sialic acid-binding adhesin of Streptococcus gordonii DL1. Infect Immun 70, 1209-1218.

Takahashi, Y., Yajima, A., Cisar, J. O. \& Konishi, K. (2004). Functional analysis of the Streptococcus gordonii DL1 sialic acid-binding adhesin and its essential role in bacterial binding to platelets. Infect Immun $\mathbf{7 2}$, 3876-3882. 
Takahashi, Y., Takashima, E., Shimazu, K., Yagishita, H., Aoba, T. \& Konishi, K. (2006). Contribution of sialic acid-binding adhesin to pathogenesis of experimental endocarditis caused by Streptococcus gordonii DL1. Infect Immun 74, 740-743.

Takamatsu, D., Bensing, B. A., Cheng, H., Jarvis, G. A., Siboo, I. R., Lopez, J. A., Griffiss, J. M. \& Sullam, P. M. (2005). Binding of the Streptococcus gordonii surface glycoproteins GspB and Hsa to specific carbohydrate structures on platelet membrane glycoprotein Ib $\alpha$. Mol Microbiol 58, 380-392.

Takamatsu, D., Bensing, B. A., Prakobphol, A., Fisher, S. J. \& Sullam, P. M. (2006). Binding of the streptococcal surface glycoproteins GspB and Hsa to human salivary proteins. Infect Immun 74, 1933-1940.

Tanzer, J. M., Grant, L., Thompson, A., Li, L., Rogers, J. D., Haase, E. M. \& Scannapieco, F. A. (2003). Amylase-binding proteins A (AbpA) and $\mathrm{B}(\mathrm{AbpB})$ differentially affect colonization of rats' teeth by Streptococcus gordonii. Microbiology 149, 2653-2660.

Tomás Carmona, I., Diz Dios, P. \& Scully, C. (2007). Efficacy of antibiotic prophylactic regimens for the prevention of bacterial endocarditis of oral origin. J Dent Res 86, 1142-1159.
Troffer-Charlier, N., Ogier, J., Moras, D. \& Cavarelli, J. (2002). Crystal structure of the V-region of Streptococcus mutans antigen I/II at $2.4 \AA$ resolution suggests a sugar preformed binding site. J Mol Biol 318, 179-188.

Wizemann, T. M., Moskovitz, J., Pearce, B. J., Cundell, D., Arvidson, C. G., So, M., Weissbach, H., Brot, N. \& Masure, H. R. (1996). Peptide methionine sulfoxide reductase contributes to the maintenance of adhesins in three major pathogens. Proc Natl Acad Sci U S A 93, 79857990.

Xiong, Y. Q., Bensing, B. A., Bayer, A. S., Chambers, H. F. \& Sullam, P. M. (2008). Role of the serine-rich surface glycoprotein GspB of Streptococcus gordonii in the pathogenesis of infective endocarditis. Microb Pathog 45, 297-301.

Xu, P., Alves, J. M., Kitten, T., Brown, A., Chen, Z., Ozaki, L. S., Manque, P., Ge, X., Serrano, M. G. \& other authors (2007). Genome of the opportunistic pathogen Streptococcus sanguinis. J Bacteriol 189, 3166-3175.

Edited by: R. J. Lamont 